\title{
Applying Artificial Neural Networks to Monitor Deposition Rate of Roll-to-roll Sputtering System in Real Time
}

\author{
Jiun-Shen Chen ${ }^{1}$ and Tzong-Daw $\mathrm{Wu}^{2}$ \\ ${ }^{1}$ Physics Division, Institute of Nuclear Energy Research, Atomic Energy Council, Executive Yuan, \\ Taoyuan City 32546, Taiwan (R.O.C) \\ ${ }^{2}$ Physics Division, Institute of Nuclear Energy Research, Atomic Energy Council, Executive Yuan, \\ Taoyuan City 32546, Taiwan (R.O.C) \\ Corresponding author's email: jschen [AT] iner.gov.tw
}

\begin{abstract}
A back propagation neural network $(B P N N)$ is applied to determine the deposition rate of a roll-toroll magnetron sputtering system in real time. Because the transmittance spectra of thin films are highly related to their thicknesses, the spectrum is a function of the thickness. Thus, determining deposited thickness through the functions is possible. However, these functions are not simple one-to-one functions; solving the inverse function to find thicknesses from spectra is difficult. Therefore, BPNNs are introduced to build approximate functions of spectra and output thicknesses. They are trained with various spectra which correspond to different film thicknesses, and will have abilities to estimate thicknesses of thin films. In this study, the estimation error of BPNNs was less than 0.6\%. The results of low error and real-time response make BPNNs a promising method for monitoring a deposition process.
\end{abstract}

Keywords- Deposition Rate, Magnetron Sputtering, Neural Networks

\section{INTRODUCTION}

For manufacturing optical thin films, the control of film thickness is essential because their optical properties highly depend on the thicknesses. Thus, monitoring and stabilizing the manufacturing process are the key points of producing high quality thin films. In this study, back propagation neural networks (BPNNs) are introduced to monitor the deposition rate of a roll-to-roll sputtering system which was designed for producing optical thin films.

In order to maintain the deposition rate of sputtering, frequent measuring is necessary. Traditionally, measuring the deposition rate of a roll-to-roll sputtering system is to attach a piece of silicon wafer or other substrates in the chamber and then measure the thickness of the deposited film after sputtering. Some advent methods are applied for determining thicknesses such as using Alpha-Step by mechanical approach directly or fitting the optical properties by software like FilmTek and Essential Macleod to estimate the thicknesses indirectly. However, all the methods above require breaking the manufacturing process to take out the deposited substrates. They are not only time-consuming but also change the conditions of manufacturing.

Optical emission spectroscopy (OES) provides another method to monitor the stability of deposition. Unlike the methods mentioned above, it monitors the intensity of some particular plasma species in real time [1,2]. In other words, it monitors the density of a particular ion to identify the stability of deposition rate immediately by assuming that they are related to each other. Deposition rate, however, depends not only on ion densities but also various sputtering conditions such as vacuum pressure, temperature and energy of charged particles [3-5]. Monitoring the stability of deposition solely by OES is limited.

As the optical properties are highly dependent on the thickness of thin films, transmittance spectra imply the information of thicknesses. Nevertheless, the transmittance spectra of thin films are complex. Only considering the light absorptions of the materials is not sufficient. Interferences of films are also need to be considered. In other words, the transmittance of thin films depends on their optical constants, i.e. the refraction index and the extinction coefficient [6].

Fitting thicknesses from the transmittance spectra via using optical equations is a new option, Lee et al. have used admittance diagram to find thickness excellently [7,8]. However, artificial neural networks (ANNs) provide another choice to estimate thicknesses without applying optical equations, such as making predictions based on coating 
parameters [9] or reflectance data [10]. In this study, BPNNs which are one kind of ANN were used to determine the thickness by learning the relationship between transmittance spectra and film thicknesses. Shown in Figure 1 is a typical feedforward neural network with two hidden layers, and the corresponding function is described as

$$
y_{i}=\sum_{r=1}^{n_{h_{2}}} w_{i r} \sigma\left(\sum_{s=1}^{n_{h_{1}}} v_{r s}^{(2)} \sigma\left(\sum_{j=1}^{m} v_{s j}^{(1)} x_{j}+\beta_{s}^{(1)}\right)+\beta_{r}^{(2)}\right), \quad i=1,2, \ldots, l
$$

Where $\mathrm{x}$ and $\mathrm{y}$ are the values of input and output respectively, $\beta$ is bias value of hidden neurons, $\mathrm{w}, \mathrm{v}$ are weights for output and hidden layers respectively, and $\sigma$ is the activation function of neurons in hidden layers [11]. In this study, the activation function of hidden neurons is a sigmoid function as follows:

$$
\sigma(t)=\frac{1}{1+e^{-t}}
$$

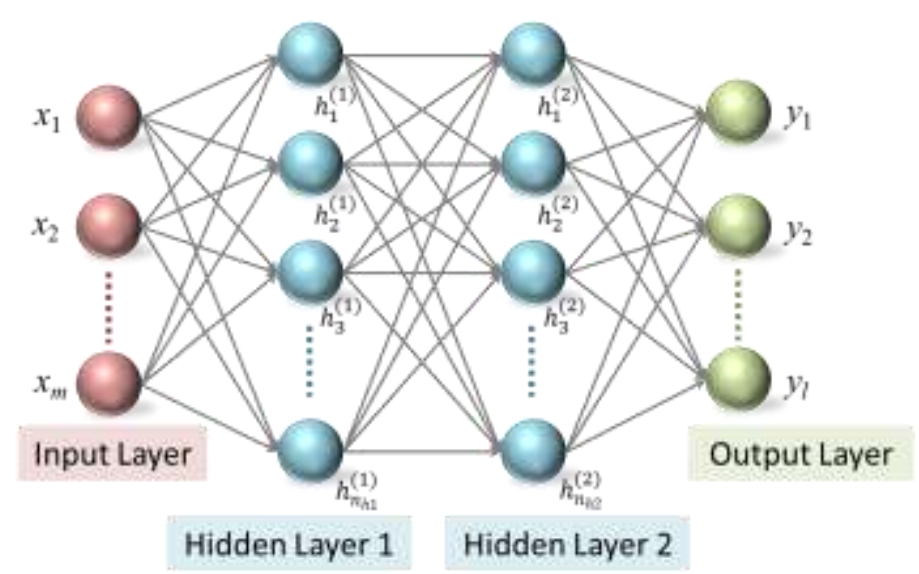

Figure 1: A Typical Feedforward Neural Network with Two Hidden Layers.

By adjusting $\mathrm{w}, \mathrm{v}$ and $\beta$, the neural network has the potential to simulate any continuous function, and the back propagation method can be used for finding these parameters. Thus, BPNNs were introduced to build an approximate function of spectrum because that thickness has some kind of relation with transmittance. In this study, various transmittance spectra of different thicknesses were used to train BPNNs, and then BPNNs can have the ability to estimate thicknesses from transmittance spectra. These BPNNs were also applied to measuring the sputtering deposition rate at different plasma power and gas flows.

\section{METHODS}

The experimental system is a roll-to-roll magnetron sputtering system equipped with a balanced deuterium halogen source (DH-2000-BAL), a spectra monitor and process control system (EMICON MC) which detection range is 200 $1100 \mathrm{~nm}$ with $1.4 \mathrm{~nm}$ resolution, and a computer that analyzed the transmittance spectra to estimate the deposited thicknesses in real time by running BPNNs. Figure 2 shows the layout of the system. 


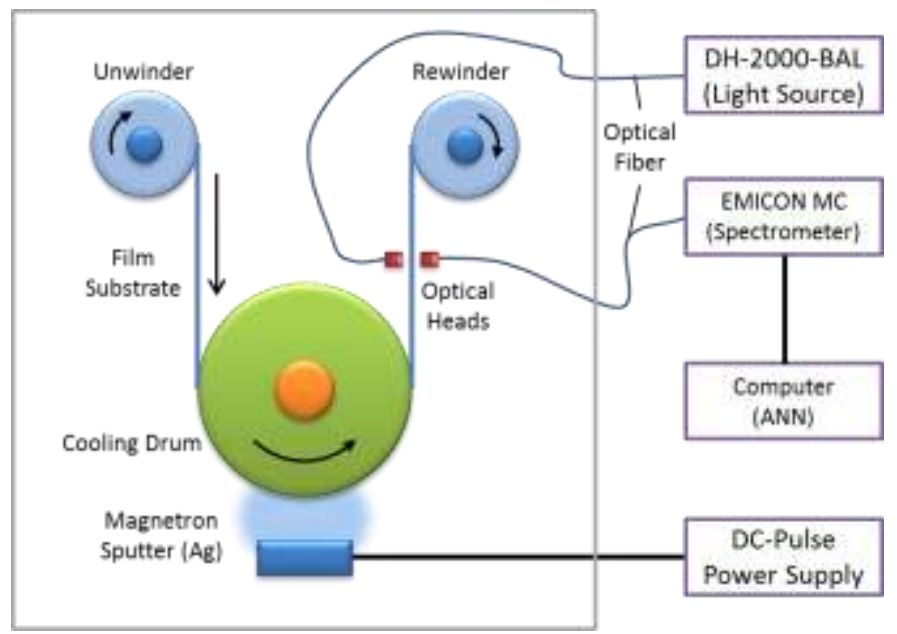

Figure 2: The Roll-to-roll Magnetron Sputtering and Monitoring System.

The target and substrate used here were silver (Ag) and polyethylene terephthalate (PET). EMICON MC is set to take transmittance spectra and send to the computer every $100 \mathrm{~ms}$. The pulsed DC power of $350 \mathrm{kHz}$ was applied to the magnetron cathode at power from 734 to $1227 \mathrm{~W}$, and the mass flow controller (MFC) for the system controlled the flow rate of argon (Ar) from 100 to $500 \mathrm{sccm}$ and hydrogen (H2) at $12 \mathrm{sccm}$. The background pressure of the chamber was $5.5 \times 10^{-6}$ torr, and the process pressure was around $3 \times 10^{-3}$ torr.

The Artificial Neural Network (ANN) used in this experiment was a BPNN, one kind of supervised neural network, and Levenberg-Marquardt (LM) algorithm was applied for the training process. Figure 3 illustrates the architecture of the BPNN which were one input layer, one or two hidden layers, and one output layer. The input layer accepted a transmittance spectrum whose wavelength range was from $320 \mathrm{~nm}$ to $1000 \mathrm{~nm}$, and the output layer gave us the estimated thickness of the $\mathrm{Ag}$ thin film. The activation function of neurons placed in hidden layers was sigmoid and in output layer was linear.

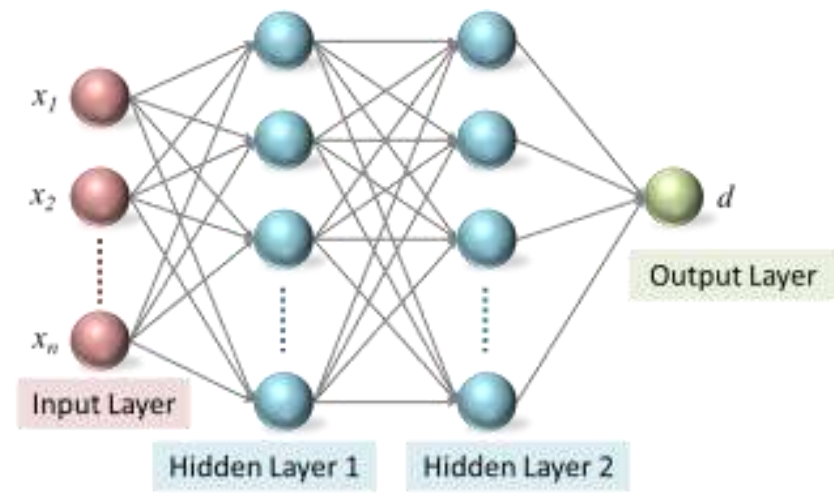

Figure 3: Architecture of the ANN. The input set $\left\{x_{i}\right\}$ and the output d represent the transmittance spectrum and film thickness respectively.

The BPNN played as a function of a spectrum, and thickness d could be found through this function.

$$
d=f\left(\left\{x_{i}\right\}\right)
$$

Where the set $\left\{x_{i}\right\}$ means a transmittance spectrum and the element $x_{i}$ represents a transmittance at specified wavelength.

\section{RESULTS}

To train the BPNNs, various transmittance spectra corresponding to different thicknesses of Ag films are required. The spectra and thicknesses are the input and learning target for the BPNNs respectively. The training spectra were prepared from transmittance spectra of thin films that were deposited on PET at different substrate speed with constant deposition rate. In a roll-to-roll system, the deposition rate is defined as deposited thickness at unit substrate speed, so the thicknesses can be described as the following equation. 
In this study, 21 groups of spectra were taken, in which 16 groups were used for training and five groups for verifying. Each group is presented by one thickness and contained 15 transmittance spectra that would be averaged as input data (as $\left\{x_{i}\right\}$ in Eq. 3) for the BPNNs.

In order to find out a suitable architecture of the BPNN, six different architectures were tested. They were one hidden layer or two hidden layers, and each hidden layer contained 5, 10, or 20 neurons. These BPNNs were trained via training data at first, and then verified against verifying data. The results are shown in Table 1 and Table 2.

Table 1: The training and verifying results of single hidden layer architectures.

\begin{tabular}{cccccc}
\hline $\begin{array}{c}\text { Wavelength Range } \\
{[\mathbf{n m}]}\end{array}$ & $\begin{array}{c}\text { Interval } \\
{[\mathbf{n m}]}\end{array}$ & $\begin{array}{c}\text { Number of Input } \\
\text { Neurons }\end{array}$ & $\begin{array}{c}\text { Number of Hidden } \\
\text { Neurons }\end{array}$ & $\begin{array}{c}\text { Training } \\
\text { Error }_{\text {avg }}\end{array}$ & $\begin{array}{c}\text { Verifying } \\
\text { Error }_{\text {avg }}\end{array}$ \\
\hline \multirow{2}{*}{$320-1000$} & \multirow{2}{*}{5} & \multirow{2}{*}{137} & 5 & $0.19 \%$ & $0.68 \%$ \\
\cline { 3 - 5 } & & & 10 & $0.18 \%$ & $0.53 \%$ \\
\cline { 3 - 5 } & & & 20 & $0.14 \%$ & $0.52 \%$ \\
\hline
\end{tabular}

Table 2: The training and verifying results of two hidden layers architectures.

\begin{tabular}{|c|c|c|c|c|c|c|}
\hline \multirow{2}{*}{$\begin{array}{l}\text { Wavelength Range } \\
{[\text { [nm] }}\end{array}$} & \multirow{2}{*}{$\begin{array}{c}\text { Interval } \\
{[\mathrm{nm}]}\end{array}$} & \multirow{2}{*}{$\begin{array}{l}\text { Number of Input } \\
\text { Neurons }\end{array}$} & \multicolumn{2}{|c|}{$\begin{array}{c}\text { Number of Hidden } \\
\text { Neurons }\end{array}$} & \multirow{2}{*}{$\begin{array}{l}\text { Training } \\
\text { Error }_{\text {avg }}\end{array}$} & \multirow{2}{*}{$\begin{array}{l}\text { Verifying } \\
\text { Error }_{\text {avg }}\end{array}$} \\
\hline & & & $1^{\text {st }}$ Layer & $2^{\text {nd }}$ Layer & & \\
\hline \multirow{3}{*}{$320-1000$} & \multirow{3}{*}{5} & \multirow{3}{*}{137} & 5 & 5 & $0.22 \%$ & $0.99 \%$ \\
\hline & & & 10 & 10 & $0.12 \%$ & $0.81 \%$ \\
\hline & & & 20 & 20 & $0.14 \%$ & $0.67 \%$ \\
\hline
\end{tabular}

The average error is defined as

$$
\text { Error }_{\text {avg }}=\frac{1}{n} \sum_{i=1}^{n}\left|\frac{T_{i}-O_{i}}{T_{i}}\right| \times 100 \%
$$

Where $\mathrm{T}_{\mathrm{i}}$ and $\mathrm{O}_{\mathrm{i}}$ represent the thickness which is the target for learning and the output of the i-th group respectively.

According to the result, the average errors of these architectures were all less than $1 \%$, thus the performances of them were remarkable. The best architecture among them was the one which had one hidden layer with 20 neutrons, and was chosen for the following experiments. Table 3 and Table 4 show the training and verifying details of this architecture respectively.

Table 3: The training details of the architecture which had one hidden layer with 20 neurons.

\begin{tabular}{|c|c|c|c|}
\hline Substrate Speed [m/min] & Thickness [nm] & $\begin{array}{l}\text { ANN Output Thickness } \\
{[\mathrm{nm}]}\end{array}$ & Error \\
\hline 1.0 & 22.39 & 22.38425117 & $0.026 \%$ \\
\hline 1.2 & 18.65833 & 18.658831 & $0.003 \%$ \\
\hline 1.4 & 15.99286 & 15.9947084 & $0.012 \%$ \\
\hline 1.6 & 13.99375 & 13.9931316 & $0.004 \%$ \\
\hline 1.8 & 12.43889 & 12.43678051 & $0.017 \%$ \\
\hline 2.0 & 11.195 & 11.1896432 & $0.048 \%$ \\
\hline 2.2 & 10.17727 & 10.18035404 & $0.030 \%$ \\
\hline
\end{tabular}




\begin{tabular}{cccc}
\hline 2.4 & 9.329167 & 9.326688334 & $0.027 \%$ \\
\hline 2.6 & 8.611538 & 8.607285 & $0.049 \%$ \\
\hline 2.8 & 7.996429 & 7.99294972 & $0.044 \%$ \\
\hline 3.0 & 7.463333 & 7.4620964 & $0.017 \%$ \\
\hline 3.2 & 6.996875 & 6.989238 & $0.109 \%$ \\
\hline 3.4 & 6.585294 & 6.577810449 & $0.114 \%$ \\
\hline 3.6 & 6.219444 & 6.213149926 & $0.101 \%$ \\
\hline 3.8 & 5.892105 & 5.895309 & $0.054 \%$ \\
\hline 4.0 & 5.5975 & 5.602231584 & $0.085 \%$ \\
\hline
\end{tabular}

Table 4: The verifying details of the architecture which had one hidden layer with 20 neurons.

\begin{tabular}{cccc}
\hline Substrate Speed $[\mathbf{m} / \mathbf{m i n}]$ & Thickness $[\mathbf{n m}]$ & $\begin{array}{c}\text { ANN Output Thickness } \\
{[\mathbf{n m}]}\end{array}$ & Error \\
\hline 1.1 & 20.3545 & 20.35804 & $0.015 \%$ \\
\hline 1.3 & 17.2231 & 17.19445 & $0.166 \%$ \\
\hline 1.5 & 14.9267 & 14.98108 & $0.362 \%$ \\
\hline 1.7 & 13.1706 & 13.21534 & $0.337 \%$ \\
\hline 1.9 & 11.7842 & 11.8494322 & $0.555 \%$ \\
\hline
\end{tabular}

The error in Table 3 and Table 4 is defined as

$$
\text { Error }=\left|\frac{T-O}{T}\right| \times 100 \%
$$

$\mathrm{T}$ and $\mathrm{O}$ represent values of the thickness and the BPNN output respectively.

When a BPNN was fully trained, it was applied to estimate the thicknesses of films in the sputtering system, and the deposition rate could be also monitored in real time since the substrate speed was controlled.

In the following experiment, an application of the BPNN was demonstrated by monitoring the deposition rate while changing the sputtering conditions. The deposition rate of a sputtering system is influenced by sputtering conditions such as intensity of plasma and pressure of a chamber. If we could determine the dependence of deposition rate on sputtering conditions, we could stabilize the film growth by adjusting sputtering conditions. However, the dependence is not identical in various sputtering systems; it is related to the design of the system. Rapid determination of the relationship will be valuable for deposition process. Hence, we applied a previously trained BPNN to monitor the deposition rate while changing the power input and the argon (Ar) flow. Both of them are important sputtering parameters, and they influence the deposition rate. Table 5 reveals the result. Figure 4 shows the deposition rate as a linear function of power input, from 734 to $1227 \mathrm{~W}$, and also illustrates that the deposition rate is proportional to Ar flow in the limited flow range, from 150 to $400 \mathrm{sccm}$. The 3D plot in Figure 5 shows an overall view of the relationship between the deposition rate, the input power and the Ar flow. This information is valuable for film growth processing. By tuning these processing parameters, the thicknesses of thin films can be controlled

It is significant to note that the table 5 contains 99 data of measuring thicknesses of thin films. By using a BPNN, all of them were measured continuously without breaking the vacuum to take the samples out from the chamber. Each datum only consumed few milliseconds for determination. 
Table 5: The deposition rate of the roll-to-roll magnetron sputter at various power input and Ar flow.

\begin{tabular}{|c|c|c|c|c|c|c|c|c|c|c|}
\hline \multirow{2}{*}{\multicolumn{2}{|c|}{$\begin{array}{l}\text { Deposition } \\
\text { rate } \\
{[\mathrm{nm} \cdot \mathrm{m} / \mathrm{min}]}\end{array}$}} & \multicolumn{9}{|c|}{ Ar Flow [sccm] } \\
\hline & & 100 & 150 & 200 & 250 & 300 & 350 & 400 & 450 & 500 \\
\hline \multirow{11}{*}{$\begin{array}{c}\text { Power } \\
{[W]}\end{array}$} & 734 & 15.9871 & 15.9696 & 15.9945 & 15.9908 & 16.0136 & 15.8713 & 15.8733 & 15.3633 & 14.8184 \\
\hline & 783 & 16.9935 & 17.2272 & 17.2332 & 17.3119 & 17.3431 & 17.2962 & 17.2902 & 16.8094 & 16.1926 \\
\hline & 832 & 17.9789 & 18.3512 & 18.3697 & 18.4326 & 18.5270 & 18.5113 & 18.5313 & 18.1092 & 17.5236 \\
\hline & 882 & 19.0224 & 19.4247 & 19.5192 & 19.5818 & 19.6949 & 19.6459 & 19.7247 & 19.3346 & 18.8261 \\
\hline & 933 & 20.0906 & 20.5308 & 20.6174 & 20.6681 & 20.7695 & 20.8336 & 20.9064 & 20.5153 & 20.0965 \\
\hline & 980 & 21.0839 & 21.5848 & 21.6314 & 21.7691 & 21.8735 & 21.9446 & 22.0039 & 21.7209 & 21.2826 \\
\hline & 1028 & 22.0785 & 22.6387 & 22.7258 & 22.8535 & 22.9771 & 23.0057 & 23.0633 & 22.7719 & 22.4443 \\
\hline & 1077 & 23.0880 & 23.7282 & 23.7946 & 23.9207 & 24.0693 & 24.1024 & 24.1078 & 23.7952 & 23.4812 \\
\hline & 1126 & 24.0962 & 24.8505 & 24.8612 & 24.9783 & 25.1558 & 25.1847 & 25.1961 & 24.8941 & 24.6005 \\
\hline & 1176 & 25.1654 & 25.7857 & 25.9077 & 26.0882 & 26.1854 & 26.2708 & 26.3328 & 25.9658 & 25.6447 \\
\hline & 1227 & 26.2062 & 26.8797 & 27.0502 & 27.1182 & 27.2598 & 27.3347 & 27.3455 & 27.0702 & 26.7323 \\
\hline
\end{tabular}

(a)

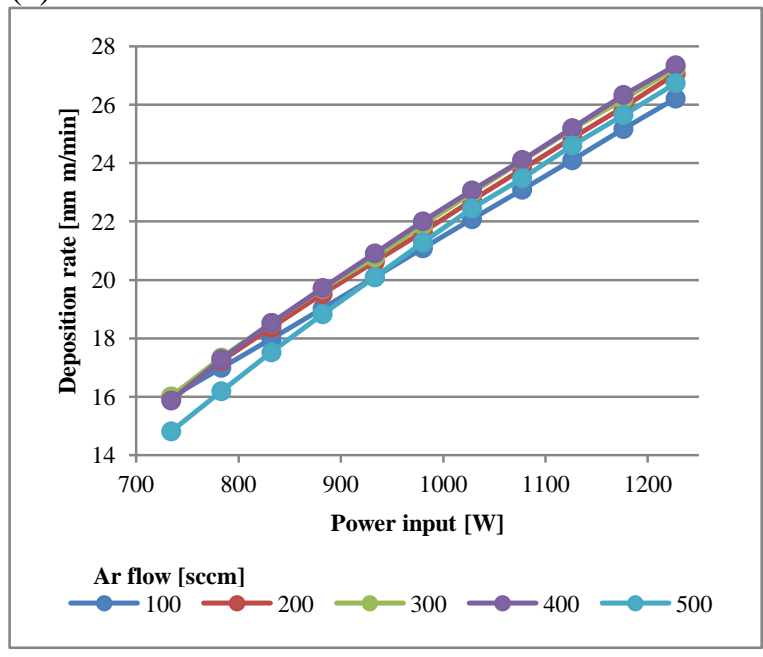

(b)

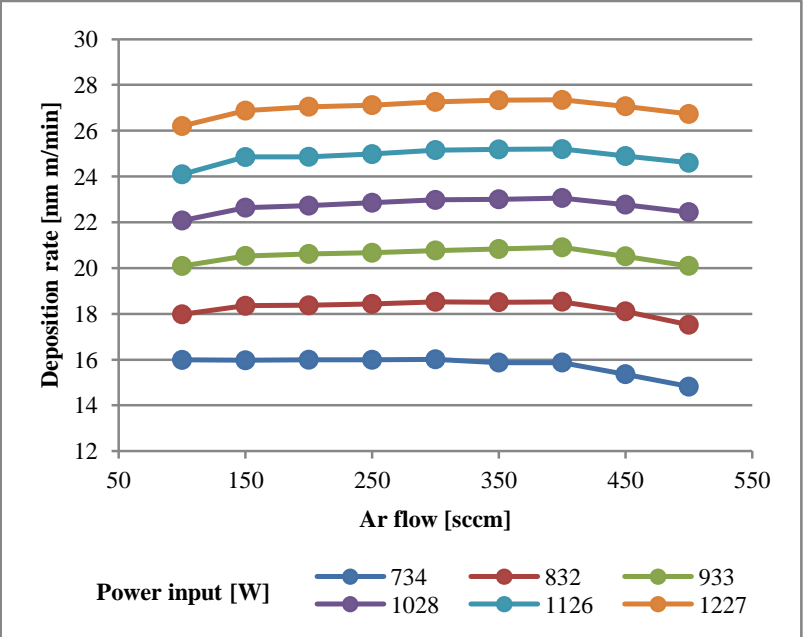

Figure 4: (a) The deposition rate versus power input. (b) The deposition rate versus Ar flow.

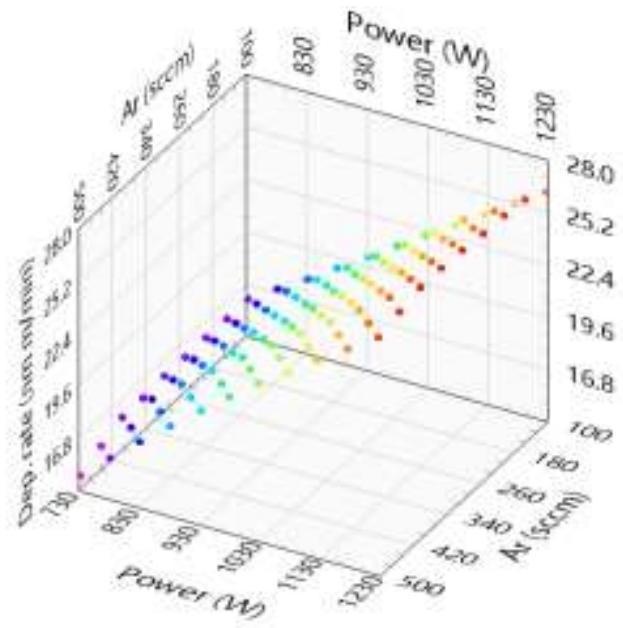

Figure 5: The deposition rate as a function of power input and Ar flow. These data were all measured in real time by the BPNN. 


\section{CONCLUSION}

In this study, a back propagation neural network (BPNN) is applied to determine the deposition rate of a roll-to-roll magnetron sputtering system in real time. The estimation error of BPNNs was less than $0.6 \%$. The results of low error and real-time response make BPNNs be a potential promising method for monitoring a deposition process. There are several advantages to determine the deposition rate of a roll-to-roll sputtering system by using BPNN. BPNN is a quick and real-time approach, and avoids intervening in the deposition processes. These features are of great importance for monitoring sputtering systems to stabilize manufacturing processes when connecting to feed-back controllers. It is especially suitable for monitoring optical thin film growth as optical properties are measured directly.

\section{ACKNOWLEDGEMENT}

The authors would like to thank Physics Division, Institute of Nuclear Energy Research, Atomic Energy Council, Executive Yuan, Taiwan for providing financial support for this research.

\section{REFERENCES}

[1] Eisgruber, I. L., et al., "Intelligent process control of indium tin oxide sputter deposition using optical emission spectroscopy", Journal of Vacuum Science \& Technology A, AVS, vol. 17, pp. 190-197, 1999.

[2] Bruns, S., et al., "High rate deposition of mixed oxides by controlled reactive magnetron-sputtering from metallic targets", Thin Solid Films, Elsevier, vol. 520, pp. 4122-4126, 2012.

[3] Kersten, H., et al., "The energy balance at substrate surfaces during plasma processing”, Vacuum, Elsevier, vol. 63, pp. 385-431, 2001.

[4] Shaginyan, L. R., et al., "Novel model for film growth based on surface temperature developing during magnetron sputtering", Surface \& Coatings Technology, Elsevier, vol. 202, pp. 486-493, 2007.

[5] Nyaiesh, A. R., Holland, L., "The dependence of deposition rate on power input for dc and rf magnetron sputtering", Vacuum, Elsevier, vol. 31, pp. 315-317, 1981.

[6] Macleod, H. A., Thin-Film Optical Filters, fourth ed., CRC Press, USA, 2010.

[7] Lee, C.-C., Chen, Y.-J., "Multilayer coatings monitoring using admittance diagram", Optics Express, OSA, vol. 16, pp. 6119-6124, 2008.

[8] Wu, K., Lee, C.-C., Ni, T.-L., "Advanced broadband monitoring for thin film deposition through equivalent optical admittance loci observation”, Optics Express, OSA, vol. 20, pp. 3883-3889, 2012.

[9] Khalaj, G., "Artificial neural network to predict the effects of coating parameters on layer thickness of chromium carbonitride coating on pre-nitrided steels”, Neural Comput \& Applic, Springer, vol. 23, pp. 779-786, 2013.

[10] Tabet, M. F., McGahan, W. A., "Use of artificial neural networks to predict thickness and optical constants of thin films from reflectance data", Thin Solid Films, Elsevier, vol. 370, pp. 122-127, 2000.

[11] Suykens, J. A. K., Vandewalle, J. P. L., De Moor, B. L. R., Artificial Neural Networks for Modelling and Control of Non-Linear Systems, Kluwer Academic Publishers, Netherlands, 1996. 